\section{La reprogrammation métabolique des carcinomes hépatocellulaires $\beta$-caténine mutés}

\section{Au cour de leur transformation}

Nadia Senni ${ }^{1-3}$, Mathilde Savall ${ }^{1-3}$, Pascale Bossard ${ }^{1-3}$

\section{Au commencement était Warburg}

La reprogrammation métabolique est un acteur majeur de la transformation tumorale [1]. Elle implique une altération ou un détournement de voies métaboliques aux dépens des voies physiologiquement actives dans la cellule saine. Cette modification du métabolisme est la résultante d'un ensemble de facteurs environnementaux et génétiques permettant à la cellule tumorale de synthétiser ses propres macromolécules tout en maintenant un statut bioénergétique et un état d'oxydo-réduction (redox) compatible avec sa survie et sa prolifération. II a été admis pendant des décennies que le métabolisme tumoral était fondé sur une utilisation accrue du glucose dans une voie non oxydative (l'effet «Warburg »), dans le but de produire des dérivés carbonés pour la synthèse des macromolécules constituantes de la cellule [2] $(\rightarrow)$.

Cette forte utilisation du glucose par

$(\rightarrow)$ Voir la Synthèse de M. Cordier-Bussat et al., $m / s n^{\circ} 8-9$, août-septembre 2018, page 701

les cellules malignes est à la base de la détection des tumeurs à l'aide de la tomographie par émission de positons au 18-fluoro-déoxyglucose (TEP-scan FDG). 0.H. Warburg ${ }^{1}$ expliquait le métabolisme glycolytique des tumeurs par l'absence d'une respiration mitochondriale fonctionnelle [3]. Or, dès les années 1920, H.G. Crabtree avait décrit une activité respiratoire bien présente dans ces cancers,

${ }^{1}$ Otto Heinrich Warburg (1883-1979), médecin lauréat du prix Nobel de physiologie ou médecine en 1931.

variable selon les tumeurs, suggérant qu'il n'y avait pas qu'un seul métabolisme tumoral, mais plutôt différentes activités métaboliques selon le type de tumeur [4]. Il est alors apparu, au cours de cette dernière décennie, que le concept de reprogrammation métabolique dépassait l'effet Warburg et qu'il existait réellement une très forte hétérogénéité métabolique selon le type de tumeur, avec une grande variété de substrats énergétiques, mais également avec diverses voies permettant la génération de biomasse.

Dans le domaine de l'oncologie, la médecine actuelle se veut de plus en plus personnalisée avec le développement d'approches thérapeutiques spécifiques à chaque type de cancer. Ces approches se fondent principalement sur les propriétés distinctives des cellules tumorales et, au cours des dernières années, de nombreuses molécules ciblant spécifiquement le métabolisme de ces cellules ont été inclues dans des essais cliniques [5]: identifier précisément le profil métabolique des différentes tumeurs est alors indispensable à la mise en place de tels outils thérapeutiques.

\section{Le carcinome hépatocellulaire}

Le cancer primitif du foie, ou carcinome hépatocellulaire ( $\mathrm{CHC}$ ), est de mauvais pronostic et constitue la deuxième cause de mortalité liée au cancer au monde. Le pronostic de cette tumeur reste sombre avec une survie médiane de 5 à 7 mois. Le Sorafenib² est à l'heure

\footnotetext{
${ }^{2}$ Un inhibiteur de kinases multiples.
}

${ }^{1}$ Inserm, U1016, Institut Cochin, 24, rue du faubourg Saint-Jacques, 75014 Paris, France. ${ }^{2}$ Cnrs, UMR8104, Paris, France.

${ }^{3}$ Université Paris Descartes, Sorbonne Paris Cité, Paris, France.

pascale.bossard@inserm.fr

actuelle la seule molécule de thérapie ciblée pour les CHC, mais son effet est limité : la survie du patient n'est allongée que de quelques mois supplémentaires. Il est donc indispensable de développer de nouvelles stratégies thérapeutiques permettant de cibler plus efficacement ces tumeurs.

Une classification des CHC réalisée à partir d'approches génomiques a permis de mettre en évidence deux grands sousgroupes de cancers hépatocellulaires : un sous-groupe enrichi en tumeurs avec une forte instabilité chromosomique, présentant, entre autres, des mutations de TP53 ou d'AXINI codant respectivement le suppresseur de tumeur p53 et l'axine 1 , et un deuxième sous-groupe de tumeurs présentant une faible instabilité chromosomique et des mutations activatrices du gène codant la $\beta$-caténine, CTNNBI (ou CHC CTNNB1) [6]. Les tumeurs CTNNBl, qui présentent une hyper-activation anormale de la voie $\beta$-caténine, sont moins prolifératives, plus différenciées, et présentent surtout un morphotype métabolique particulier, puisque, contrairement aux autres classes de CHC, elles ne sont jamais stéatosées ${ }^{3}$. Elles sont le plus souvent indétectables par TEP-Scan FDG. Ces différences de morphotype entre tumeurs suggèrent une reprogrammation métabolique distinctive selon la classe de CHC. Nous avons entrepris d'identifier ces altérations métaboliques afin d'aider au développement

${ }^{3}$ La stéatose est définie par l'accumulation d'acides gras sous forme de triglycérides dans le cytoplasme des hépatocytes. 


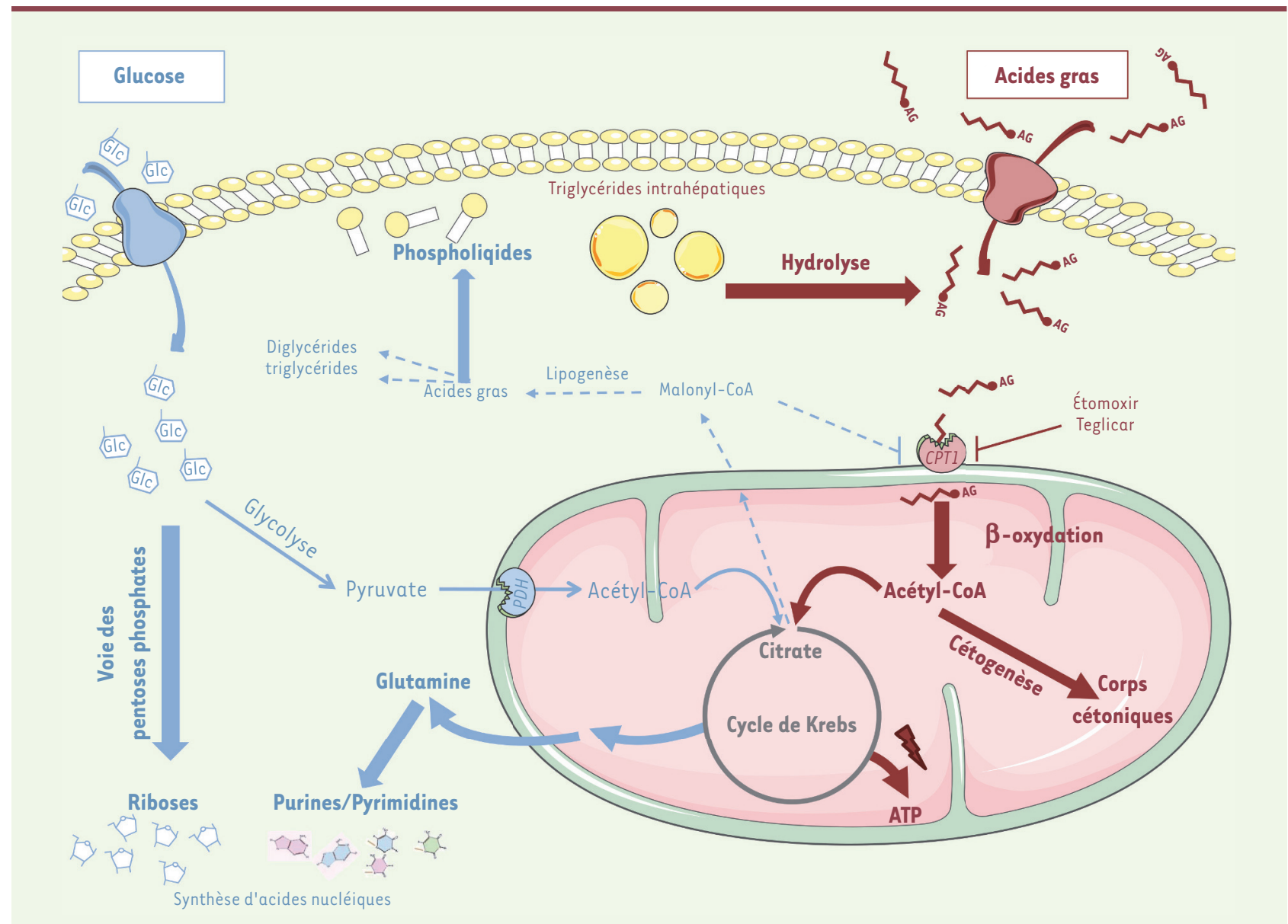

Figure 1. Reprogrammation métabolique des hépatocarcinomes exprimant la $\boldsymbol{\beta}$-caténine activée. Suite à l'activation oncogénique de la voie $\beta$-caténine, l'oxydation des acides gras est accrue et cette voie métabolique peut être ciblée afin de ralentir la progression tumorale. En rouge : métabolisme des acides gras, en bleu, métabolisme du glucose, en vert, les inhibiteurs de l'oxydation lipidique ayant été inclus dans des essais cliniques. L'Étomoxir est un inhibiteur allostérique irréversible de toutes les isoformes de la CPTl et le Teglicar est spécifique de l'isoforme hépathique. En gras, voies métaboliques stimulées, en pointillés, voies métaboliques réprimées. CHC CTNNBI : hépatocarcinome exprimant la $\beta$-caténine activée ; CPTl : carnitine palmitoyltransférase I; PDH : pyruvate déshydrogénase.

d'outils diagnostiques et thérapeutiques les ciblant spécifiquement [6].

\section{Oublions Warburg...}

À l'aide de modèles murins mimant la tumorigenèse hépatique dépendant de la $\beta$-caténine (CHC $\beta$-caténine activée), nous avons montré que l'activation oncogénique de cette voie de signalisation conduisait dans le foie au développement de tumeurs présentant une reprogrammation métabolique très particulière, puisque s'affranchissant de l'effet Warburg. Ces tumeurs ne sont pas glycolytiques: comparativement aux tissus sains, elles ne présentent pas de différence dans l'oxydation du glucose, ni d'altération dans la production de lactate, un marqueur de la glycolyse [6]. Mais, même si les cellules saines et tumorales semblent utiliser le glucose de façon similaire, il s'avère que le devenir de ce dernier diffère entre les deux types cellulaires. Dans le tissu tumoral, une majeure partie des carbones du glucose est en fait réorientée vers la synthèse de pentoses phosphates, génératrice de riboses, et vers une production massive de glutamine, donneur principal d'amines pour la synthèse des bases puriques et pyrimidiques. Ces mécanismes permettent donc de générer les acides nucléiques nécessaires à la prolifération sans pour autant mettre en jeu l'effet Warburg. En revanche, la génération d'ATP à partir du glucose est fortement diminuée, suggérant que ces tumeurs utilisent une autre source d'énergie [7].

\section{Et brûlons nos acides gras !}

Dans un foie sain, à l'état post-absorptif $^{4}$, la principale source d'énergie est assurée par l'oxydation mitochondriale des acides gras (ou $\beta$-oxydation). À l'état post-prandial ${ }^{5}$, cette voie est réprimée. $0 r$, dans les $\mathrm{CHC} \beta$-caténine activée, la $\beta$-oxydation est fortement, et surtout constamment, induite, quelque-

\footnotetext{
${ }^{4}$ Juste après la prise alimentaire.

${ }^{5}$ Après la digestion.
} 
soit l'état nutritionnel. Dans ces tumeurs, cette oxydation accrue est alors le principal pourvoyeur d'ATP via les oxydations phosphorylantes, ce qui confère aux cellules des avantages prolifératifs. Cette hyperactivation de la $\beta$-oxydation n'a pas été retrouvée dans un modèle murin de $\mathrm{CHC}$ ne présentant pas d'activation de la $\beta$-caténine. Dans une cohorte de patients présentant un $\mathrm{CHC}$, cette activation de la $\beta$-oxydation n'est également présente que dans les CHC CTNNBI mutés [7]. II semblerait donc que la reprogrammation métabolique, observée dans les CHC $\beta$-caténine activée, soit un reflet direct de cet oncogène CTNNBl sur le métabolisme. Mais par quel mécanisme? L'activation de la voie $\beta$-caténine dans les foies murins et humains est en fait associée à l'induction du facteur de transcription PPAR $\alpha$ (peroxisome proliferator-activated receptor alpha), un acteur majeur du contrôle génique de la $\beta$-oxydation. Ce facteur de transcription est alors le médiateur des effets de la $\beta$-caténine sur l'oxydation lipidique puisque, dans un modèle murin où l'expression de PPAR $\alpha$ est invalidée, l'activation de la voie $\beta$-caténine ne conduit pas à une hyperactivation de la $\beta$-oxydation.

\section{L'oxydation lipidique : talon d'Achille des carcinomes hépatocellulaires $\boldsymbol{\beta}$-caténine activée}

Cette reprogrammation métabolique précéderait la transformation tumorale, et semble nécessaire à la transformation des CHC $\beta$-caténine activée [7]. Chez les souris n'exprimant pas PPAR $\alpha$ (par invalidation du gène) et présentant une $\beta$-oxydation hépatique beaucoup moins active, l'initiation et la progression tumorale des CHC $\beta$-caténine sont très fortement diminuées, avec une incidence tumorale 5 fois plus faible. Traiter les CHC $\beta$-caténine activée dès leur initiation, avec l'étomoxir, un inhibiteur pharmacologique de la $\beta$-oxydation, s'est par ailleurs révélé efficace pour ralentir très fortement leur progression. Une hyperactivation de la $\beta$-oxydation est donc indispensable à la transformation et à la progression des CHC $\beta$-caténine.

\section{Utiliser les caractéristiques} métaboliques des CHC $\beta$-caténine activée pour leur diagnostic et leur traitement

Le développement de méthodes diagnostiques fiables est un enjeu majeur en cancérologie. Parmi les méthodes non invasives, le TEP-Scan, utilisant des sondes ciblant une voie métabolique spécifique, permet de distinguer un tissu tumoral d'un tissu sain sur la base de son activité métabolique augmentée. Récemment, de nouvelles sondes ont émergé, comme le 3- ${ }^{18}$ fluoro-2,2 dimethylpropionic acid qui permet la détection de l'activité de la $\beta$-oxydation [8]. Actuellement, cette sonde a été utilisée et validée pour la détection de certains cancers du sein. Elle pourrait être aussi proposée pour la détection des CHC CTNNBI.

La plasticité tumorale est l'un des principaux obstacles dans le traitement par des inhibiteurs métaboliques. Les cellules cancéreuses peuvent modifier leur métabolisme au cours du développement tumoral, ou en réponse à des traitements ciblant une voie métabolique particulière, devenant ainsi résistantes aux drogues utilisées. Mais les CHC $\beta$-caténine activée possèdent une certaine rigidité métabolique puisque la diminution de la $\beta$-oxydation est suffisante pour prévenir leur initiation et ralentir leur progression. L'utilisation d'un traitement étant dictée par la balance entre bénéfices et risques, une modulation contrôlée de la $\beta$-oxydation pourrait être prise en considération dans le cas des CHC CTNNB1, limitant ainsi les dommages collatéraux sur les tissus sains environnants. Une thérapie ciblée pour cette classe de CHC est alors parfaitement envisageable. Lors d'essais cliniques, l'étomoxir, un inhibiteur allostérique irréversible de toutes les isoformes de la CPTl (la carnitine palmitoyltransférase, première enzyme de la $\beta$-oxydation), s'est avéré cytotoxique pour les tissus sains [9]. Plus récemment, un inhibiteur réversible de la CPTl, spécifique de l'isoforme hépatique, le Tegiclar, est entré en phase II d'un essai clinique pour le traitement de pathologies associées au diabète de type 2 [10]. Cet inhibiteur s'est également avéré efficace dans des tests précliniques pour le traitement de lymphomes à forte activité de $\beta$-oxydation. Il pourrait donc être envisagé comme traitement des CHC CTNNB1. Que ce soit pour un diagnostic ou une approche thérapeutique, la dépendance aux acides gras des CHC CTNNBI pourrait être une faiblesse exploitable. $\diamond$ $\beta$-catenin mutated hepatocarcinoma metabolic rewiring at the heart of their transformation

\section{LIENS D'INTÉRÊT}

Les auteurs déclarent n'avoir aucun lien d'intérêt concernant les données publiées dans cet article.

\section{RÉFÉRENCES}

1. Hanahan D, Weinberg RA. Hallmarks of cancer: the next generation. Cell $2011 ; 144: 646-74$.

2. Cordier-Bussat M, Thibert C, Sijobert P, et al., Même l'effet Warburg est oxydable- Coopération métabolique et développement tumoral. Med Sci (Paris) $2018 ; 34: 701-8$.

3. Warburg 0 , Wind $F$, Negelein $\varepsilon$. The metabolism of tumors in the body. J Gen Physiol 1927 ; $8: 519-30$.

4. Crabtree HG. Observations on the carbohydrate metabolism of tumours. Biochem J $1929 ; 23: 536-45$.

5. Mathupala SP. Metabolic targeting of malignant tumors: small-molecule inhibitors of bioenergetic flux. Recent Pat Anticancer Drug Discov 2010 ; 6 : 6-14.

6. Zucman-Rossi J, Benhamouche S, Godard C, et al. Differential effects of inactivated Axinl and activated beta-catenin mutations in human hepatocellular carcinomas. Oncogene 2007 ; 26 : 774 80.

7. Senni N, Savall M, Cabrerizo Granados D, et al. Beta-catenin-activated hepatocellular carcinomas are addicted to fatty acids. Gut 2018; gutjnl-2017-315448. doi: 10.1136/ gutjnl-2017-315448.

8. Witney TH, Pisaneschi F, Alam IS, et al. Preclinical evaluation of 3-18F-fluoro-2,2-dimethylpropionic acid as an imaging agent for tumor detection. J Nucl Med 2014 ; 55 : 1506-12.

9. Holubarsch CJ, Rohrbach M, Karrasch M, et al. A double-blind randomized multicentre clinical trial to evaluate the efficacy and safety of two doses of etomoxir in comparison with placebo in patients with moderate congestive heart failure: the ERGO (etomoxir for the recovery of glucose oxidation) study. Clin Sci (Lond) 2007 ; 113 : 205-12.

10. Conti R, Mannucci $\varepsilon$, Pessotto P, et al. Selective reversible inhibition of liver carnitine palmitoyltransferase 1 by teglicar reduces gluconeogenesis and improves glucose homeostasis. Diabetes $2011 ; 60 \mathrm{~b}$ : 644-51. 